\title{
Peripheral blood leukocyte counts as a marker of severity of functional lung impairment in OAD patients attending a tertiary care hospital in Manipur, India
}

\author{
Ashem Nandarani Devi*1 ${ }^{1}$, W. Kanan Devi ${ }^{2}$, W. Asoka Singh ${ }^{3}$, Kanmi \\ Ningshen ${ }^{1}$, AwungshiJannie Shimray ${ }^{1}$, Hongprachan Hungyo ${ }^{1}$ \\ I. Postgraduate students, Department of Physiology, RIMS, Manipur, ${ }^{2}$ Professor, Department of Physiology, \\ RIMS, Manipur, ${ }^{3 .}$ Professor and HOD, Department of Respiratory Medicine, RIMS, Manipur, India
}

\begin{abstract}
:
Background: Systemic inflammation is associated with impaired lung function and inflammation is a part of Obstructive Airway Disease (OAD). Chronic obstructive pulmonary disease (COPD) and bronchial asthma are the two most common $O A D$. In view of this, the present study was done with the objective of determining the relationship between the various grades of severity of airway obstruction in COPD and asthma with blood leukocyte counts.

Methods: A cross-sectional study enrolling 40 COPD \& 25 asthmatic patients of both sexes. Lung function was studied by computerized spirometer (Helios 402) and blood leukocyte counts (absolute neutrophil count, absolute eosinophil count, absolute basophil count, absolute lymphocyte count and absolute monocyte count) were determined by using suitable diluting fluids.

Results: Grading of COPD and asthma severity was done according to GOLD and GINA guidelines respectively based on FEV $V_{1} \%$ predicted. With the increase in severity of airway obstruction, FEV1 was found to decline in both the groups. Out of 40 COPD patients, $12.5 \%$ of patients had mild, $20 \%$ had moderate, $40 \%$ had severe and $27.5 \%$ had very severe COPD. Out of 25 asthma patients, $48 \%$ had mild, 32\% had moderate and $20 \%$ had severe asthma. There was a significant increase in neutrophil count with the increase in COPD severity and with the increase in asthma severity, eosinophil count increases significantly. . Monocyte count was also increased with the decline in lung function in both the groups.

Conclusion:Peripheral blood leukocyte counts are useful and can be used as an indirect \& inexpensive marker of severity of functional lung impairment in OAD patients.
\end{abstract}

Keywords: OAD, COPD, asthma, spirometer, FEV1\% predicted, FEV1, leukocyte counts

\section{Introduction}

Obstructive diseases of the airways are a diverse group of respiratory diseases. In general, they result from narrowing of any portion of the airways (from upper airway to bronchioles less than $2 \mathrm{~mm}$ in diameter) and finally resulting in a reduction of maximal airflow in relation to maximal flow, with the consequent increase in the work of breathing. ${ }^{(1)}$ Included in the obstructive pulmonary disorders are chronic obstructive pulmonary disease of the airways (chronic bronchitis and emphysema), asthma, bronchiectasis, small airway disease, and upper airway obstruction. ${ }^{(2)}$

The 2011 revision of the Global Initiative for Asthma (GINA) guidelines defines asthma as a chronic inflammatory disorder of the airways in which many cells and cellular elements play a role, in particular, mast cells, eosinophil, T-lymphocytes, macrophages, neutrophils and epithelial cells ${ }^{(3)}$.The 2011 revision of the GOLD guidelines defines COPD (Chronic Obstructive Pulmonary Disease) as a preventable and treatable disease which is characterized by persistent airflow limitation that is usually progressive and associated with an enhanced chronic inflammatory response in the airways and the lung to noxious particles or gases. ${ }^{(4)}$ Neutrophils, eosinophil, alveolar macrophages, and lymphocytes all appear to participate in the inflammatory process associated with COPD. ${ }^{(5)}$

The gold standard definition of obstructive airway disease is Forced Expiratory Volume in $1 \mathrm{sec}$ (FEV1) and ratio of FEV1 to Forced Vital Capacity (FEV1/FVC) less than fifth percentile. ${ }^{(6)}$ The level of FEV1 is also used to grade the severity of airway obstruction. ${ }^{(7)}$

Assessment of ongoing inflammation is often determined by evidence of inflammatory cells in respiratory tract. The present study was undertaken to explore whether circulating levels of leukocytes in blood bear any relationship with the severity of the disease as reflected in the functional lung impairment assessed by spirometry. 


\section{Study design}

\section{Materials And Methods}

This is a cross-sectional study carried out in the Department of Physiology and Department of Respiratory Medicine, Regional Institute of Medical Sciences (RIMS), Manipur, India, during the period from November 2012 to December2013. After obtaining approval from the institutional ethics committee and written informed consent, a total of 65 OAD patients of both sexes between the ages of 18 to 65 years who attended Respiratory Medicine OPD and ward were enrolled in the study.

\section{Exclusion criteria}

Patients with associated diseases like worm infestations, atopic skin disease and other allergic diseases, cardiac problems, renal failure, diabetes mellitus, hypertension, pulmonary fibrosis, neuromuscular diseases, and ascites were excluded.

\section{Data collection procedure}

A) Spirometric studies were conducted by means of a Computerized Spirometer- Helios 402 of the Recorders and Medicare system, Chandigarh. The study variables recorded by spirometry include Forced Vital Capacity (FVC), Forced Expiratory Volume in one second $\left(\mathrm{FEV}_{1}\right), \mathrm{FEV}_{1} / \mathrm{FVC}$, Peak Expiratory Flow Rate (PEFR), Forced Expiratory Flow ( $\left.\mathrm{FEF}_{25-75 \%}\right)$.

1) Classification of severity of airflow limitation in COPD patients according to GOLD guidelines- Based on post bronchodilator $\mathrm{FEV}_{1}$

(In patients with $\mathrm{FEV}_{1} / \mathrm{FVC}<0.70$ )

GOLD 1 Mild $\quad \mathrm{FEV}_{1} \geq 80 \%$ predicted

GOLD 2Moderate $\quad 50 \% \leq \mathrm{FEV}_{1}<80 \%$ predicted

GOLD 3 Severe $\quad 30 \% \leq \mathrm{FEV}_{1}<50 \%$ predicted

GOLD 4 Very severe $\quad \mathrm{FEV}_{1}<30 \%$ predicted

2) Classification of severity of airflow limitation in Asthma patients according to GINA guidelines-

Mild $\quad \mathrm{FEV}_{1} \geq 80 \%$ predicted

Moderate $\quad 60 \% \leq \mathrm{FEV}_{1}<80 \%$ predicted

Severe $\quad \mathrm{FEV}_{1}<60 \%$ predicted

B) Assessment of blood leukocyte counts was done by using $1 \mathrm{ml}$ of blood collected by venipuncture from the subjects. The count was made under a compound microscope in an Improved Neubauer's chamber using Turk's fluid and Leishman's stain. After finding out Total Leukocyte Count(TLC) and Differential Leukocyte Counts(DLC), Absolute Leukocyte Counts was determined by using the formula-

Absolute leukocyte count $=$ DLC $/ 100 \times \mathrm{TLC}$

\section{Statistical analysis}

Appropriate statistical analysis like ANOVA was made wherever necessary, using standard software (Microsoft Excel 2007 and SPSS version 16.0). Level of statistical significance was set atp-value $<0.05$.

\section{Results}

Grading of COPD and asthma severity was done according to GOLD and GINA guidelines respectively based on $\mathrm{FEV}_{1} \%$ predicted. Out of 40 COPD patients, $12.5 \%$ of patients had mild, $20 \%$ had moderate, $40 \%$ had severe and $27.5 \%$ had very severe COPD. Out of 25 asthma patients, $48 \%$ had mild, $32 \%$ had moderate and $20 \%$ had severe bronchial asthma.

Table 1: Socio-demographic profile of the patients of COPD and asthma groups

\begin{tabular}{|lll|}
\hline Parameters & COPD & Bronchial asthma \\
\hline No. of subjects & 40 & 25 \\
Age (mean) in years & $54.69 \pm 16.65$ & $32.38 \pm 18.09$ \\
Male: Female & $22: 18$ & $11: 14$ \\
Type of residence, $\mathrm{n}(\%)$ & Urban=32(80) & Urban= 18(72) \\
Rural=8(20) & Rural=7(28) & \\
Smoking status, $\mathrm{n}(\%)$ & Non-smoker=10(25) & Non-smoker=12(48) \\
Smoker=18(45) & Smoker=5(20) & \\
Ex-smoker=12(30) & Ex-smoker=8(32) & \\
\hline
\end{tabular}

The above table shows the socio-demographic distribution of the patients. There are more male cases than female cases in COPD group whereas more female cases in asthma group. The patients in the asthma group are younger than the COPD groups. Maximum of the study population resides in the urban areas in both 
the groups. With regards to smoking habit, maximum of the COPD cases are smoker whereas non-smoker in asthma cases.

Table 2: Mean \pm SD of FEV1 in different grades of severity of COPD and bronchialasthma

\begin{tabular}{|c|c|c|c|c|}
\hline OAD & Mild & Moderate & Severe & Very severe \\
\hline COPD & $96.14 \pm 21.18$ & $57.75 \pm 9.61$ & $40.5 \pm 10.96$ & $18.37 \pm 5.60$ \\
\hline Asthma & $80.37 \pm 13.01$ & $68.12 \pm 4.38$ & $48 \pm 11.64$ & 0.079 (NS) \\
\hline
\end{tabular}

( $\mathrm{S}=$ statistically significant, $\mathrm{NS}=$ statistically not significant)

The above table shows that as the severity of airway obstruction increases, FEV1 decreases in both the groups which werestatistically not significant.

Table 3:Comparison of mean \pm SEM of absolute leukocyte counts in different grades of $\underline{\text { COPD }}$ severity

\begin{tabular}{|llllll|}
\hline ParametersMild & \multicolumn{1}{c}{ Moderate } & Severe & Very severe & p-value & \\
\hline ANC & $4617 \pm 331.8$ & $6339 \pm 690.2$ & $6512.2 \pm 198.9$ & $8667.3 \pm 174.1$ & $0.042(\mathrm{~S})$ \\
AEC & $182.6 \pm 63.5$ & $274.9 \pm 62.04$ & $200.8 \pm 55.7$ & $215.4 \pm 58.8$ & $0.741(\mathrm{NS})$ \\
ALC & $2276.6 \pm 170.1$ & $2245.1 \pm 96.16$ & $1801.8 \pm 62.5$ & $2003.5 \pm 79.8$ & $0.209(\mathrm{NS})$ \\
AMC & $202.4 \pm 67.34$ & $291.7 \pm 21.10$ & $308.1 \pm 72.60$ & $384.1 \pm 48.21$ & $0.192(\mathrm{NS})$ \\
\hline
\end{tabular}

$(\mathrm{ABC}=0$ in all the grades of $\mathrm{COPD})$

(ANC: Absolute neutrophil count, AEC: Absolute eosinophil count, ABC: Absolute basophil count, ALC:

Absolute lymphocyte count, AMC: Absolute monocyte count)

The above table shows a significant increase in neutrophil count as the severity of airway obstruction increases in COPD group. Monocyte count also increases with the increase in severity. No significant changes in lymphocyte and eosinophil counts.

Table 4:Comparison of mean \pm SEM of absolute leukocyte counts in different grades of bronchial asthma

\begin{tabular}{|lllcc|}
\hline Parameters Mild & & Moderate & Severe & \multicolumn{1}{c|}{ p- value } \\
\hline ANC & $3281.5 \pm 212.9$ & $4526.6 \pm 290.6$ & $4677.5 \pm 460.0$ & $0.059(\mathrm{NS})$ \\
AEC & $309.3 \pm 43.6$ & $381.2 \pm 50.3$ & $441.6 \pm 156.12$ & $0.032(\mathrm{~S})$ \\
ALC & $1933.6 \pm 230.23$ & $2106.7 \pm 186.12$ & $2086.2 \pm 615.8$ & $0.847(\mathrm{NS})$ \\
AMC & $313.6 \pm 130.4$ & $630.4 \pm 128.2$ & $635.3 \pm 226.6$ & $0.317(\mathrm{NS})$ \\
\hline
\end{tabular}

$(\mathrm{ABC}=0$ in all the grades of asthma)

The above table shows a significant increase in eosinophil count with the increase in severity of airway obstruction in asthma group. Neutrophil and monocyte counts also increases with increase in the severity. No significant changes in lymphocyte count.

\section{Discussion}

The present study was done to explore whether the circulating levels of leukocytes in blood bear any relationship with the severity of the disease as reflected in the functional lung impairment assessed by spirometry. The study was based on the primary data collected from 40 COPD and 25 Bronchial Asthma patients.

Based on GOLD and GINA guidelines, grading of severity of functional lung impairment of COPD and bronchial asthma respectively was done based on $\mathrm{FEV}_{1} \%$ predicted. In COPD group, maximum patients $(40 \%)$ presented with severe COPD and maximum asthma patients $(48 \%)$ presented with mild severity.

In our study, increased neutrophil count in COPD patients was observed. Similar finding was reported in a study conducted by Rathod VPS et al ${ }^{(8)}$. The neutrophil count was also increased with the fall in FEV1. Similar findings were reported in other studies ${ }^{(9,10)}$. It is well known that neutrophils play a crucial role in the pathophysiology of COPD, as they release multiple mediators and tissue degrading enzymes such as elastases that orchestrate tissue destruction and chronic inflammation. In COPD the neutrophilic inflammatory response dominates.

Other reasons of high neutrophil count in increased severity may be the presence of respiratory infection and use of steroids for treatment as severity of functional lung impairment increases. Steroids causes an increase in neutrophil count by accelerating release from bone marrow and decreasing its migration out of circulation

Monocytes were predominantly associated with symptoms indicative of obstructive airway disease, in similar relation to neutrophils, but both of these leukocyte counts were also increased in asthma patients. In our study, we also found an increase in monocyte count in both COPD and asthma groups. Similar results were seen in a study conducted by Sarah AL etal ${ }^{(11)}$.

The present study found a higher eosinophil count in bronchial asthma patients and the count increases as the severity increases. Similar findings were also observed in a study conducted by Horn BR etal ${ }^{(12)}$. Asthma 
was classically considered an allergic disease with an eosinophilic inflammatory picture but in the last decade another phenotype of asthma has been defined, non-allergic asthma characterized by neutrophilicinflammation (13).

Cigarette smoking is the major factors in the development of obstructive airways disease. Investigators have studied a variety of potential mechanisms by which cigarettesmoke might alter protease-antiprotease balance in the lung. In our study, majority of COPD patients were smoker $(45 \%)$ which might have contributed to the increased neutrophil count in COPD patients. This was in conformity with the study conducted by Willemse BWM et al ${ }^{(14)}$.

Neutrophil numbers are also increased in bronchoalveolar lavage fluids from cigarette smoker ${ }^{(15)}$.

\section{Conclusion}

Our study shows that a relationship exists between blood leukocyte counts and severity of functional lung impairment in COPD and asthma patients (OADs). We have found a significant association between blood neutrophil and eosinophil counts with increase in COPD and asthma severity respectively. These data suggest that the peripheral leukocyte counts can serve as an indirect marker of severity of functional lung impairment in Obstructive Airway Disease patients.

However, smoking status, presence of respiratory infections, and use of bronchodilators and inhaled corticosteroids for treatment can influence the results. Further studies of the pathophysiologic mechanisms by which peripheral leukocyte counts are related with the decline in lung function involving more number of patients in different hospitals seems indicated.

\section{References}

[1]. Nadel JA. Obstructive diseases: General Principles and Diagnostic Approach. In: Murray JF, Mason RJ, Boushey Jr HA, Nadel JA, editors. Textbook of Respiratory Medicine. $3^{\text {rd }}$ Ed. Philadelphia: WB Saunders Company; 2000. p. 1173-184.

[2]. Grippi MA, Tino G. Pulmonary Function Testing. In: Fishman AP, Elias JA, Fishman JA, Grippi MA, Sevior RM, Pack AI, editors. Fishman's Pulmonary Diseases and Disorders. $4^{\text {th }}$ Ed, Vol 1, USA: McGraw Hill; 2007.p. 602.

[3]. Global Initiative for asthma (GINA): Global strategy for asthma management and prevention. 2011. Available from: http://www.ginaasthma.org. Accessed on May 7, 2012.

[4]. Global initiative for chronic obstructive lung disease. Global strategy for the diagnosis, management, and prevention of COPD (revised 2011). 2011. Available from: http://www.goldcopd.org. Accessed on October 12, 2012.

[5]. Snider GL. Understanding Inflammation in Chronic Obstructive Pulmonary Disease. Am J Respir Crit Care Med. 2003; 167: 104549.

[6]. Straus SE, McAlister FA, Sackett DL, Deeks JJ. The Accuracy of Patient History, Wheezing, and Laryngeal Measurements in Diagnosing Obstructive Airway Disease. JAMA. 2000; 283(14): 1853-57.

[7]. Pride NB. Spirometry and Mechanics - Test of Forced Expiration and Inspiration. In: Hughes JMB, Pride NB, editors. Lung Function Tests - Physiological Principles and Clinical appiclations. $1^{\text {st }}$ Ed. Hong Kong: W B Saunders Company; 1999 . p.3-25

[8]. Rathod VPS, Kapoor P, Pillai KK, Khanam R. Assessment of asthma and chronic obstructive pulmonary disorder in relation to reversibility, IgE, eosinophil, and neutrophil count in a University Teaching Hospital in South Delhi, India. J Pharm Bioall Sci. 2010; 4:337-40.

[9]. Wouters EF, Reynaert NL, Dentener MA, et al. Systemic and local inflammation in asthma and chronic obstructive pulmonary disease: is there a connection? Proc Am Thorac Soc. 2009; 6:638- 47.

[10]. Sparrow D, Glynn RJ, Cohen M, et al. The relationship of the peripheral leukocyte count and cigarette smoking to pulmonary function among adult men. CHEST. 1984; 86:383-86.

[11]. Sarah AL, Ian DP, John RS,Alan JK, Scott TW, John RB, The Relation Between Peripheral Blood Leukocyte Counts and Respiratory Symptoms, Atopy, Lung Function, and Airway Responsiveness in Adults. CHEST. 2001; 119:105-14

[12]. Horn BR, Robin ED, Theodore J, Vankessel A. Total Eosinophil counts in the management of Bronchial Asthma. N Engl J Med. 1975 May; 292(22): 1152-55.

[13]. Gibson PG, Simpson JL, Saltos N. Heterogeneity of airway inflammation in persistent asthma: evidence of neutrophilic inflammation and increased sputum interleukin-8. CHEST. 2001; 119:1329-36.

[14]. Willemse BWM, Postma DS , Timens W, ten Hacken NHT.The impact of smoking cessation on respiratory symptoms, lung function, airway hyper responsiveness and inflammation. Eur Respir J. 2004 March; 23(3): 464-76.

[15]. Huninghake GW, Crystal RG. Cigarette smoking and lung destruction. Accumulation of neutrophils in the lungs cigarette smokers. Am Rev Respir Dis. 1983; 128:833-41. 